\title{
An Investigation of the Role of Working Memory Capacity and Naming Speed in Phonological Advance Planning in Language Production
}

\author{
Jana Klaus ${ }^{1}$ \\ Herbert Schriefers \\ Radboud University \\ Producing multi-word utterances is a complex, yet relatively effortless process. Research with the \\ picture-word interference paradigm has shown that speakers can plan all elements of such \\ utterances up to the phonological level before initiating speech, yet magnitude and polarity differ \\ between but also within studies. We investigated possible sources for variability in the \\ phonological advance planning scope. In two experiments, participants produced bare nouns \\ ("monkey") and complex noun phrases ( "the small red monkey") while ignoring distractor words \\ phonologically (un)related to the noun. For low- and high-working memory capacity speakers as \\ well as fast and slow speakers, we found phonological facilitation effects for the bare noun, but \\ no distractor effects for the complex noun phrases. However, looking at individual distractor \\ effects for utterance-final elements revealed a large variability between speakers. We conclude \\ that phonological advance planning cannot be summarised as an overall effect, but should take \\ into account inter- and intraindividual variability.
}

Keywords: language production; working memory; phonological advance planning

\section{Introduction}

Speaking constitutes a unique human ability, in which to-be-uttered concepts are encoded grammatically and phonologically to be articulated. The fact that we do this in a fluent manner has led researchers to assume that several words are planned ahead before we initiate speech. However, the size of this advance planning scope is still debated. Previous research has established that on the syntactic-lexical level, speakers can - and usually do - plan up until the final element of a multi-word utterance (i.e., complex noun phrases and simple sentences; e.g., Meyer, 1996; Schriefers, 1992; Schriefers, de Ruiter, \& Steigerwald, 1999; Wagner, Jescheniak, \& Schriefers, 2010; cf. Wheeldon, Ohlson, Ashby, \& Gator, 2013; Zhao \& Yang, 2016). In contrast, studies on the planning on the phonological level provide a less consistent picture. While a number of studies reported phonological activation of utterance-final elements (Costa \& Caramazza, 2002; Damian \& Dumay, 2007;
Jescheniak, Schriefers, \& Hantsch, 2003; Oppermann, Jescheniak, \& Schriefers, 2010; Schnur, 2011; Schnur, Costa, \& Caramazza, 2006), others did not obtain such an effect (Meyer, 1996; Schriefers \& Teruel, 1999; Smith \& Wheeldon, 2004). Moreover, there has been evidence that the phonological advance planning scope can vary as a function of a concurrent cognitive load (Klaus, Mädebach, Oppermann, \& Jescheniak, 2017; Oppermann et al., 2010) or as a function of individual differences (Michel Lange \& Laganaro, 2014; Schriefers \& Teruel, 1999). Overall then, this suggests that the scope of phonological advance planning is flexible, between speakers, but potentially also within speakers, and the factors influencing this variability are not yet understood.

The current study investigates the influence of inter- and intraindividual differences on phonological advance planning in multi-word utterances. Specifically, we looked at whether verbal working memory capacity (WMC;

\footnotetext{
${ }^{1}$ Correspondence concerning this article should be addressed to Jana Klaus (j.klaus@ donders.ru.nl).
} 
Experiment 1) and variations in naming latencies (Experiment 2) are predictors of phonological advance planning. Both experiments were carried out in Dutch and made use of the picture-word interference paradigm, in which participants describe a picture while ignoring auditory distractors (Schriefers, Meyer, \& Levelt, 1990), in our study auditory non-words. These distractors can be phonologically related to the target word (e.g., "aad" for the target noun "aap" [monkey]) or unrelated (e.g., “miek”). By comparing the production of bare nouns (e.g., "aap") and complex noun phrases (e.g., "de kleine rode aap” [the little red monkey]), we can directly contrast the phonological activation of the noun (e.g., "aap") in different positions of the utterance (i.e., utteranceinitial in bare noun production and utterance-final in complex noun phrase production). In bare noun production, phonologically related distractors typically facilitate the naming response compared to an unrelated condition (e.g., Damian \& Martin, 1999; Schriefers et al., 1990). For complex noun phrases (NPs) as in the current study, facilitation effects (Costa \& Caramazza, 2002; Damian \& Dumay, 2007), interference effects (Jescheniak et al., 2003), and null effects (Michel Lange \& Laganaro, 2014; Schriefers \& Teruel, 1999) have been observed. Before turning to the present experiments, we will briefly review previous research on phonological advance planning in multiword utterances. Since the current study focuses on the production of complex NPs, we will focus on studies which employed comparable utterance formats (for studies investigating phonological advance planning in sentence production, see e.g., Klaus et al., 2017; Meyer, 1996; Oppermann et al., 2010; Schnur, 2011; Schnur et al., 2006; Smith \& Wheeldon, 2004).

One of the first studies that reported utterancefinal distractor effects in complex NPs longer than two words came from Costa and Caramazza (2002). In this study, native speakers of English produced either bare nouns (e.g., “dog”), determiner-noun phrases (e.g., "the dog") or determiner-adjectivenoun phrases (e.g., "the red dog”). Distractors phonologically related to the noun (e.g., "doll”) consistently led to faster naming latencies compared to an unrelated distractor (e.g., "brick"), regardless of the position of the target noun within the utterance. Crucially, the same pattern was observed when Spanish speakers produced complex utterances combining a noun and an adjective in post-nominal (i.e., utterance-final) position (e.g., "la casa azul" [the blue house]). Again, distractors phonologically related to the utterance-final element (e.g., "azul”) sped up naming compared to an unrelated condition. The authors concluded that all elements of a complex NP are planned ahead up to the phonological level prior to speech onset.

In a similar study with German speakers, Jescheniak, Schriefers \& Hantsch (2003) examined phonological advance planning in bare nouns (e.g., "Hund" [dog]), determiner-noun phrases (e.g., "der Hund" [the dog]), and determiner-adjectiveadjective-noun phrases (e.g., "der große rote Hund" [the big red $\operatorname{dog}$ ]). Noun-related distractor words facilitated naming in the two first conditions, but led to longer naming latencies in the most complex condition at SOA 0ms. This polarity shift in the distractor effects led the authors to propose a graded activation account of phonological encoding, which will also serve as the working model for the current study. The rationale is that the amount to which elements in a complex utterance are activated phonologically before articulation decreases linearly (i.e., "from left to right"), with the earliest (leftmost) elements receiving the most and the latest (right-most) elements the least activation. This activation gradient codes the order in which the words are eventually articulated. In the production of complex NPs, a distractor related to the final element adds activation to this final element, which in turn interferes with the serial order coding of the utterance. This account thus predicts that phonological facilitation obtained in initial positions should be attenuated, or even turn into interference, when the primed element appears at a later position in the utterance. It should be noted, however, that the studies by Jescheniak et al. (2003) and Costa and Caramazza (2002) tested utterance format between participants, which implies that a direct comparison of individual advance planning processes across utterance formats was not possible.

Damian and Dumay (2007) investigated whether an increase in task demands modulates the scope of phonological advance planning. Participants produced determiner-adjective-noun phrases (e.g., "the blue carrot"), with half of the sample being required to respond within a time limit. This time limit was meant to reduce the scope of advance planning. The authors obtained facilitation effects from noun-related distractors both with and without a response deadline, leading them to conclude that the scope of phonological advance planning is not 


\section{DIFFERENCES IN PHONOLOGICAL ADVANCE PLANNING}

susceptible to increasing task demands as introduced by a response deadline. Again, however, the critical manipulation was tested between participants, which hinders the investigation of interindividual differences. Furthermore, the authors did not address potential intraindividual differences.

A study by Michel Lange and Laganaro (2014) provided some first evidence that the scope of phonological advance planning can differ between speakers. Participants produced French adjectivenoun phrases, in which the adjective appears in either pre- or post-nominal position (e.g., "grand canard" [big duck] or "balai rouge" [red broom]) while ignoring distractor words phonologically (un)related to either the adjective or the noun. If the primed element appeared in the first position, related distractors sped up naming, regardless of whether the utterance began with an adjective or a noun. For the second word, by contrast, no reliable effect was found. However, the authors could show that for trials which were initiated relatively late (around 900ms after picture onset), there was indeed a facilitation effect with respect to the second word. In a second experiment, the authors further investigated the influence of naming speed on phonological advance planning. Participants produced determiner-adjective-noun phrases while ignoring distractors related to the adjective or the noun, with the adjective always preceding the noun. To look at interindividual differences in advance planning, the participants were assigned to a fast and a slow group based on their general naming speed. Distractors related to the adjective decreased naming latencies in both speed groups relative to an unrelated condition. With regard to the utterancefinal element, however, only the slow group showed a facilitating distractor effect while the fast group showed no difference between the related and the unrelated condition. The authors concluded that "inter-subject variability can account for different encoding patterns at the level of phonological encoding in a picture naming task" (p. 9; see Wagner et al., 2010, for converging evidence on the syntactic-lexical level). Note that Schriefers and Teruel (1999) reported a similar pattern. Overall, participants producing adjective-noun phrases in German (e.g., "lila Säge”) showed no effect from distractors phonologically related to the second syllable of the first element (nor beyond this boundary), but a subsequent analysis dividing speakers into quick and more hesitant initiators - as quantified by the amount of disfluencies throughout the utterance - provided an additional finding. Participants who produced more fluent utterances showed a facilitation effect at the second syllable of the first word as well, while there was no such effect for speakers who were less fluent and thus produced more repairs.

Taken together, previous research has shown that phonological advance planning can include the utterance-final element of complex NPs. However, there is also evidence that this planning scope may be subject to interindividual differences, as manifested in different planning strategies throughout an experiment. The current study aims to investigate potential sources of these differences. In Experiment 1, we contrast participants with high and low verbal WMC. In Experiment 2, we investigate how interindividual variations in general naming speed affect phonological advance planning.

\section{Experiment 1}

Experiment 1 investigated whether interindividual differences in verbal WMC can account for the variability observed in phonological advance planning. It is generally assumed that WMC is related to interindividual differences in language comprehension (Baddeley, 2003; Daneman \& Merikle, 1996), and there is evidence from singleword naming (Shao, Roelofs, \& Meyer, 2012) and syntactic planning in subject-verb agreement production (Hartsuiker \& Barkhuysen, 2006) suggesting that WMC plays a role in language production as well. In Experiment 1, we therefore investigated the scope of phonological advance planning in speakers with high and low WMC.

It is not really obvious, however, whether a high WMC should lead to a larger or a smaller phonological advance planning scope. On the one hand, high WMC may translate into a larger advance planning scope, such that high-WMC speakers may be able to plan ahead more elements than low-WMC speakers prior to speech onset. This would be the case because high-WMC speakers are better at keeping later elements of an utterance in working memory before initiating articulation of the utterance. In this case, we would expect a clear distractor effect in complex NP production for the high-WMC group, and a much smaller effect or no effect for the low-WMC group.

By contrast, it is also possible that high-WMC speakers produce complex utterances more incrementally, i.e., that they are able to 
phonologically encode later words of an utterance while already articulating the first words of the utterance. By contrast, low-WMC speakers initiate their responses only after the entire utterance has been planned ahead. In this case, we should see a large distractor effect for complex NP production for the low-WMC group and a small effect or no effect for the high-WMC group.

Finally if verbal WMC does not affect phonological advance planning at all, there should be no differences between the two groups.

\section{Methods}

Participants. Thirty-two Dutch native speakers (three male; mean age: 22.6 years, $S D=2.8$ ) participated in exchange for course credit or monetary reimbursement. All participants had normal or corrected-to-normal vision and reported no colour blindness. Due to technical complications, one participant was replaced. All participants were selected out of a larger sample of 128 people who had participated in an online version of the reading span task (Klaus \& Schriefers, 2016) prior to the experiment. This task was chosen because, contrary to simple span tasks, it captures both the processing and the storage abilities of individuals. Out of this sample, participants whose WM scores fell in the lower or upper quartile of the distribution were invited to take part in the current study. ${ }^{1}$ We tested 16 participants in the low-WMC group (mean WM score $=.74, S D=.05)$ and 16 participants in the high-WMC group (mean WM score $=.96$, $S D=.02)$.

Materials. We used 36 black and white linedrawings of simple objects with a mono- or disyllabic name in Dutch. In the familiarisation phase, each picture was sized to fill an imaginary square of $300 \times 300$ pixels (approximately $7.5 \times 7.5$ $\mathrm{cm}$ on the screen). During the experimental phases, all pictures were presented in a specific size-colour configuration. We used four different colours (red, blue, brown, green) and two sizes (big [450 $\times 450$ pixels) vs. small $[150 \times 150$ pixels $])$. Each picture was assigned a size and a colour which was kept constant throughout the experimental blocks. Care was taken that there was no phonological onset overlap between the adjectives and the target noun (i.e., we avoided combinations like "the big blue bucket”).

For each noun, a phonotactically legal pseudoword was created which shared the first consonant or consonant cluster and adjacent vowel with the target noun and had the same syllabic and prosodic structure as the target noun. Unrelated control conditions were created by reassigning the distractors to different nouns (see Appendix A for a complete list of the materials). The auditory distractors were spoken by a female native speaker of Dutch. An additional set of four items with unrelated distractors was created for use in practice blocks and warm-up trials.

Design. The design consisted of the betweenparticipant factor WMC (low vs. high) and the within-participants factors utterance format (bare noun vs. complex NP) and phonological relatedness (phonologically related vs. unrelated to the noun). All factors were tested within items. Utterance format was blocked, and all participants began by producing the bare noun format followed by the complex NP format. This fixed order was chosen to be able to use a phonological 'baseline' effect elicited in the bare noun production block as a reference for a potential phonological effect in the complex NP production. To be usable in the same way for all participants, this baseline should be collected in the same way for all participants.

Procedure. Each participant was tested individually. They were seated comfortably in front of the computer screen. Prior to the experimental blocks, participants were familiarised with the items. All items were presented as black line drawings one by one on the screen, and participants were asked to name them spontaneously. Afterwards, the intended target name was shown on the screen, and participants were instructed to use only this name throughout the experiment. In two subsequent practice blocks consisting of 12 trials each, first the size-colour manipulation and then the distractors were introduced. Participants were instructed to ignore both the size and colour of the targets, as well as the distractor words, and to continue naming the targets with a single word (e.g., "aap" [monkey]) as quickly as possible. This was followed by the first experimental block consisting of 72 trials. Finally, another practice block (12 trials) introduced the complex NP format. Participants were now instructed to also name the size and colour of the presented targets using a complex NP (e.g., "de kleine rode aap" [the small red monkey]) while ignoring the auditory distractor words. In total, an experimental session lasted about 45 minutes.

An experimental trial was structured as follows. First, a fixation cross appeared at the centre of the screen for $250 \mathrm{~ms}$, followed by a white screen for 


\section{DIFFERENCES IN PHONOLOGICAL ADVANCE PLANNING}

$250 \mathrm{~ms}$. Then, the picture appeared at the centre of the screen and stayed there until the voice-key had registered a response, or for a maximum of $1,000 \mathrm{~ms}$. Responses were registered up to $2,500 \mathrm{~ms}$ after picture presentation. After a $250 \mathrm{~ms}$, the next trial was initiated. The auditory distractor was presented at the same time as the picture (SOA 0ms).

Analyses. Observations were discarded from the naming latency analyses whenever (a) a picture was named erroneously or not at all; (b) a speechunrelated sound preceded the target utterance and triggered the voice-key; (c) a disfluency occurred or an utterance was corrected; (d) there was a perceptible pause within the utterance; (e) a speech onset latency was shorter than $200 \mathrm{~ms}$ or longer than $3000 \mathrm{~ms}$; or (f) the voice-key was not triggered due to technical errors. All of these cases except for (e) and (f) were included in the error analyses. Observations deviating from a participant's and an item's mean by more than three standard deviations (computed by utterance format and relatedness) were marked as outliers and also removed from the naming latencies without coding an error.

Statistical analyses were computed with mixedeffects models using the lme4 package (Version 1.1.10, Bates, Mächler, Bolker, \& Walker, 2015) in $\mathrm{R}$ (Version 3.2.4; R Core Team, 2016). The factors WMC (low vs. high), utterance format (bare noun vs. complex NP), and relatedness (phonologically related vs. unrelated) were sum-coded and included as fixed effects in the models. Participants and items were included as random effects (Baayen, Davidson, \& Bates, 2008). We started all analyses with a maximal random-effects structure, that is, models including random intercepts and random slopes (for all fixed effects and their interactions) for both participants and items (Barr, Levy, Scheepers, \& Tily, 2013). Only if the model with the maximal random-effects structure did not converge, we simplified it by the stepwise removal of the higher order terms.

Naming latencies were log-transformed to normalize their distribution. Visual inspection of residual plots did not reveal deviations from homoscedasticity or normality of the transformed data. For naming latencies, we report $t$ values, which are considered significant at the $\alpha=.05$ level if their absolute value exceeds 2. Error rates were analysed using mixed logit regression (Jaeger, 2008), and Wald's $z$ and associated $p$ values are reported.

Table 1

\section{Results}

353 erroneous trials $(7.7 \%)$ and 12 outliers $(0.3 \%)$ were excluded from the naming latency analyses. Table 1 displays mean naming latencies and error rates broken down by WMC, utterance format, and relatedness, averaged across participants.

In the analysis of naming latencies, all main effects were significant. High-WMC participants responded faster than low-WMC participants (main effect WMC: $\beta=0.046, \quad S E=0.022, t=2.1$ ). Participants responded faster in the bare noun condition compared to the complex NP condition (main effect utterance format: $\beta=0.050$, $S E=0.016, \quad t=3.1)$. Phonologically related distractors sped up naming latencies compared to unrelated distractors (main effect relatedness: $\beta=0.021, S E=0.004, t=4.8$ ). There was an interaction of format and relatedness $(\beta=0.021$, $S E=0.004, t=5.2$ ), indicating that a facilitating effect from related distractors was found for the bare noun format $(\beta=0.041, S E=0.006, t=6.5$ ), but not for the complex NP format ( $\beta=0.0003, S E=0.005$, $t=0.1)$. None of the other interactions were significant (all $t \mathrm{~s} \leq 0.6$ ).

In the analysis of error rates, there was only a main effect of utterance format $(\beta=0.537$, $S E=0.062, t=8.6, p<.001)$, indicating higher error rates in the complex NP format. None of the other effects were significant (all $p \mathrm{~s}>.171$ ). To investigate whether simple distractor effects were predictive of distractor effects in the advance planning of non-initial elements, we correlated individual distractor effects (i.e., the difference between the related and the unrelated condition) of the bare noun production and the complex NP production. However, neither for the low-WMC nor for the high-WMC group, these correlations were significant (low: $r=-.23, p=.398$; high: $r=.26, p=$ $.331)$.

\section{Discussion}

Experiment 1 investigated whether individual verbal WMC as measured by a reading span task affects phonological advance planning in simple and complex utterances. Participants with high WMC responded faster than participants with low WMC, but there was no difference between these groups in terms of distractor effects: Both groups showed

Table 1 
Mean naming latencies in milliseconds and error rates in percent of Experiment 1, broken down by working memory capacity (high vs. low), utterance format (bare noun vs. complex noun phrase) and relatedness (phonologically related $v$ s. unrelated to the target noun), aggregated by participants.

\begin{tabular}{lllll}
\hline & \multicolumn{2}{c}{ High WMC } & \multicolumn{2}{c}{ Low WMC } \\
\cline { 2 - 5 } & RT in ms & Errors in \% & RT in ms & Errors in \% \\
\hline Bare noun related & $638(13)$ & $3.6(1.0)$ & $701(24)$ & $4.0(0.8)$ \\
Bare noun unrelated & $698(13)$ & $3.5(0.8)$ & $759(28)$ & $6.1(1.2)$ \\
Difference & $-59(9)$ & $0.2(1.1)$ & $-58(11)$ & $-2.1(1.3)$ \\
\hline Complex NP related & $748(40)$ & $10.4(2.2)$ & $818(35)$ & $12.0(1.6)$ \\
Complex NP unrelated & $746(39)$ & $9.4(1.8)$ & $819(35)$ & $12.3(1.9)$ \\
Difference & $2(11)$ & $1.0(1.3)$ & $-1(10)$ & $-0.3(1.4)$ \\
\hline
\end{tabular}

Note. Mean standard errors in brackets. Negative difference scores reflect phonological facilitation. RT = reaction times. $\mathrm{NP}=$ noun phrase.

phonological facilitation in the bare noun condition, but no effect in the complex NP condition. This is in contrast to previous studies which reported a phonological distractor effect for non-initial primed elements (Costa \& Caramazza, 2002; Damian \& Dumay, 2007; Jescheniak et al., 2003). However, it should be noted that the statistical null effect which ultimately averages across all collected valid responses - may also come about by a combination of both facilitating and interfering effects obtained across participants. The polarity of effects on noninitial words of complex utterances is not consistent across studies, with some studies reporting facilitation effects (Costa \& Caramazza, 2002; Damian \& Dumay, 2007; Michel Lange \& Laganaro, 2014; Schnur, 2011; Schnur et al., 2006; Schriefers \& Teruel, 1999) and others inhibition effects (Jescheniak et al., 2003; Klaus et al., 2017; Oppermann et al., 2010). Both facilitation and inhibition effects are usually considered an indicator of phonological advance planning beyond utteranceinitial elements. In terms of the graded activation model (Jescheniak et al., 2003, see also introduction) a distractor that is phonologically related to a word in a non-initial position of an utterance facilitates the phonological encoding of this word, but at the same time disturbs the coding of the serial order of words in the utterance. Thus whether the eventual neteffect is facilitation, inhibition (or even a null-effect) depends on the relative strength of these two components.

In order to examine whether the null effect for complex NPs is actually consistent across all participants, we compared the mean distractor effects for both utterances for each of the 32 participants (see Figure 1). Inspection of these phonological effects shows three main points. First, the facilitation effect for the bare noun was present in almost all participants, although it showed an interindividual range of about $160 \mathrm{~ms}$. Second, for the complex NP, the range of the distractor effect was even higher across participants, and importantly included both facilitation and interference effects. Third, in line with the graded activation account (Jescheniak et al., 2003), the slope from bare noun to complex NP effects was positive for 27 out of the

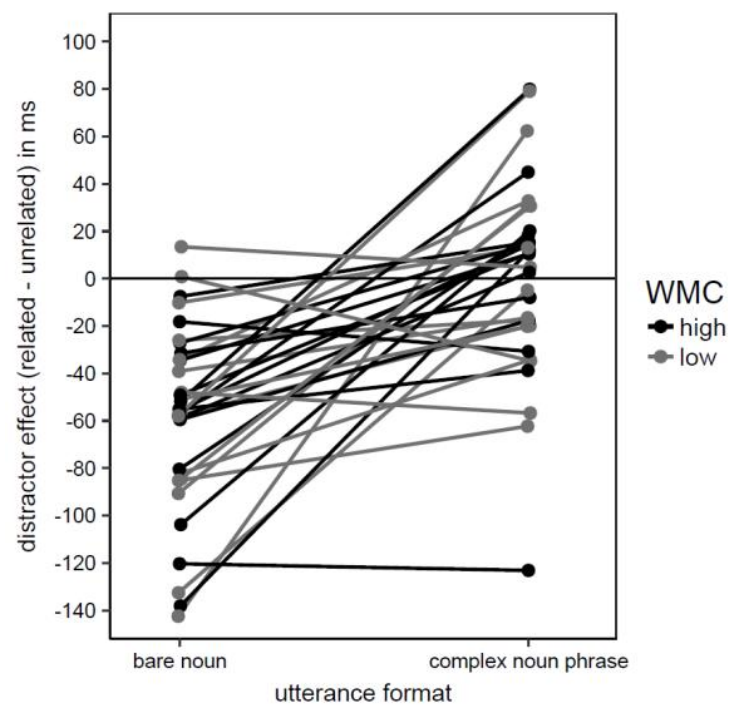

Figure 1. Individual distractor effects for the bare noun and the complex noun phrase conditions for Experiment 1, depicted for the low-and high-WMC groups. Negative values indicate facilitation from phonologically related distractors, and positive values indicate interference.

32 tested participants. That is, for the majority of the 


\section{DIFFERENCES IN PHONOLOGICAL ADVANCE PLANNING}

participants, the phonological effect obtained in the complex NP condition was indeed attenuated compared to bare noun naming. Finally, this overall pattern did not vary as a function of verbal WMC, as the variability with respect to the phonological effects in the complex NP condition was present for both WMC groups.

Thus, Experiment 1 could not establish a link between interindividual differences in verbal WMC (as determined by individual reading span) and phonological advance planning in multi-word utterances. However, looking at distractor effects on the individual level, we could show that the overall null effect with respect to the utterance-final element - both within and between WMC groups - is actually the result of a large interindividual variability including facilitation, null, and inhibition effects. This is not compatible with the conclusion that the utterance-final element had not been planned ahead at all - as the overall grand averages might suggest - because then we should see all individual effects for complex NPs clustering around $0 \mathrm{~ms}$, i.e., no difference between the related and the unrelated condition. In other words, there is reason to believe that the majority of speakers actually do plan up to the last element, but that the polarity and magnitude of the appropriate distractor effect is not consistent across speakers. We will come back to this issue in the General Discussion. In Experiment 2, we examined another potential source for interindividual differences in phonological advance planning, differences in general naming speed.

\section{Experiment 2}

As we have outlined in the Introduction, to date only two studies have examined the influence of naming speed on phonological advance planning in complex NP production using two different methodological approaches. Damian and Dumay (2007) investigated whether introducing a response deadline would reduce the phonological advance planning unit, but found no difference between effects of phonologically related distractors with and without a response deadline. Michel Lange and Laganaro (2014) used a different approach to test for potential effects of naming speed. They compared slow and fast speakers and found a distractor effect for the utterance-final element of complex NPs only for the slow group. The present experiment combines these two approaches to gain a better understanding of the potential influence of naming speed on phonological advance planning. Based on the previous studies, the predictions are quite straightforward: (1) If general naming speed (as reflected in the distinction between fast and slow speakers in bare noun production) determines the size of the advance planning scope, as the results by Michel Lange and Laganaro (2014) suggest, we should find evidence that slow speakers in our experiment plan further ahead than fast speakers when there is no time pressure. (2) Following Damian and Dumay (2007), introducing a deadline should not change this pattern of results. However, given the large interindividual variability of distractor effects for complex NPs observed in Experiment 1, we have to adjust our predictions accordingly. Increasing the time pressure by means of a response deadline should remove large parts of the variability observed in Experiment 1 because speakers' naming speed will be homogenized both at an inter- and intraindividual level. Interindividual variability will be reduced because slow speakers will be forced to adapt their naming speed, and intraindividual variability across trials (e.g., exceptionally long naming latencies caused by lapses of attention) will be reduced by excluding the possibility to respond after the deadline (see Procedure for details).

To test these hypotheses, we contrasted slow and fast speakers with respect to their phonological advance planning span for normal naming responses (i.e., when there is no response deadline) and under time pressure (i.e., in the presence of a response deadline). Experiment 2 was identical to Experiment 1 , except that we (1) did not screen for WMC because we had found no differences between high and low WMC groups in Experiment 1, (2) split the sample of 32 participants in 16 fast and 16 slow responders, and (3) added two additional blocks introducing a response deadline of $750 \mathrm{~ms}$. This deadline was chosen because it was in the range of the mean naming latencies in the unrelated conditions of the previous experiment $(729 \mathrm{~ms}$ for bare noun production and $783 \mathrm{~ms}$ for complex NP production) and should therefore implement sufficient time pressure without causing too many naming errors or time-outs.

\section{Methods}

Participants. Thirty-two native speakers of Dutch (5 male; mean age: 21.5 years, $S D=2.4$ ) participated in exchange for money or course credit. Two participants were replaced because they had 
less than $65 \%$ valid data points in the deadline blocks (due to time-outs or erroneous responses).

Materials and design. The materials were identical to those used in Experiment 1. The design included the within-participants and within-items factors utterance format (bare noun vs. complex NP), phonological relatedness (phonologically related vs. unrelated to the noun), and response deadline (with vs. without), and the betweenparticipant and within-item factor speed group (fast vs. slow). Utterance format and response deadline were blocked. All participants first produced the bare noun and the complex NP without the response deadline, followed by two blocks with the deadline (each preceded by a practice block 12 trials each). This fixed order was chosen to first provide a direct replication of Experiment 1 in the first two blocks, and subsequently investigate the effect of a response deadline on advance planning.

Procedure and analyses. The procedure was identical to that of Experiment 1, except that after the two experimental blocks without a deadline, two additional blocks including a response deadline were added. Participants were instructed that they only had a limited amount of time to respond, and they were encouraged to respond in this time window. They were also told that they were not required to respond after the time-out (i.e., after $750 \mathrm{~ms}$ ) because these responses would not be counted.

An experimental trial of the deadline blocks was structured as those of the previous blocks, except that the target picture was visible for a maximum of $750 \mathrm{~ms}$, which was also the latest time point at which the microphone would register a response. If no response had been detected by this time, participants received visual feedback on screen ( "Te langzaam!" [Too slow!]), urging them to respond faster.

The data were analysed as in Experiment 1. Trials that were not responded to within $750 \mathrm{~ms}$ in the deadline blocks were coded as time-outs and removed from the analyses without coding an error. To examine potential differences between fast and slow speakers, we split the sample in two groups based on their individual mean naming latencies in the unrelated condition when producing bare nouns without a response deadline. On average, the fast speakers responded at $609 \mathrm{~ms}$ in this condition (SD $=64$, range: 515-726), and the slow speakers at $704 \mathrm{~ms}(\mathrm{SD}=120$, range: 575-1129).

\section{Results}

358 time-outs (i.e., trials that were not initiated by the response deadline of $750 \mathrm{~ms} ; 3.9 \%), 806$ erroneous trials $(7.3 \%)$ and 10 outliers $(0.1 \%)$ were excluded from the naming latency analyses. We will report the analyses separately for the two deadline conditions because we expect differences to emerge between those two conditions, and running a mixedeffects model including a four-way interaction (including all interaction terms in the random slopes) is not meaningful.

Distractor effects without a response deadline. Table 2 displays mean naming latencies and error rates broken down by speed group, utterance format, and relatedness in the no-deadline condition. Naturally, participants responded faster in the fast group than in the slow group (main effect speed group: $\quad \beta=0.109, \quad S E=0.021, \quad t=5.3$ ). Phonologically related distractors significantly decreased naming latencies compared to unrelated distractors (main effect relatedness: $\beta=0.019$, $S E=0.003, t=5.5)$. There was an interaction between utterance format and relatedness $(\beta=0.021, \quad S E=0.004, \quad t=4.8), \quad$ indicating a significant influence of related distractors on naming latencies in the bare noun condition $(\beta=0.040, S E=0.006, t=6.4)$, but not the complex NP condition (main effect relatedness: $\beta=0.001$, $S E=0.004, t=0.3$ ). None of the other effects were significant in the analysis of naming latencies ( $t$ s < 1.48).

Participants made more errors when producing complex NPs (main effect utterance format: $\beta=0.600, S E=0.063, z=9.5, p<.001)$. Utterance format and speed group interacted $(\beta=0.164$, $S E=0.063, z=2.6, p=.009)$, indicating that the increase in error rates from the bare noun to the complex NP format was somewhat more pronounced for the slow speakers $(\beta=0.753$, $S E=0.094, z=8.0, p<.001)$ than the fast speakers $(\beta=0.429, S E=0.082, z=5.3, p<.001)$. None of the other effects were significant in the analysis of error rates $(p s>$.204). Again, the correlations between the distractor effect during bare noun naming and the distractor effect during complex NP naming were not significant for both speed groups (slow speakers: $r=.16, p=.566$; fast speakers: $r=$ $.10, p=.711)$.

Distractor effects with a response deadline. Table 3 displays mean naming latencies and error 


\section{DIFFERENCES IN PHONOLOGICAL ADVANCE PLANNING}

Table 2

Mean naming latencies in milliseconds and error rates in percent of Experiment 2 for the no-deadline condition, broken down by speed group (fast vs. slow), utterance format (bare noun vs. complex noun phrase) and relatedness (phonologically related vs. unrelated to the target noun), aggregated by participants.

\begin{tabular}{lllll}
\hline & \multicolumn{3}{c}{ Fast speakers } & \multicolumn{2}{c}{ Slow speakers } \\
\cline { 2 - 5 } & RT in ms & Errors in $\%$ & RT in ms & Errors in \% \\
\hline Bare noun related & $602(14)$ & $4.5(0.8)$ & $745(27)$ & $3.8(1.4)$ \\
Bare noun unrelated & $660(12)$ & $5.7(1.5)$ & $797(26)$ & $2.8(0.9)$ \\
Difference & $-58(10)$ & $-1.2(1.5)$ & $-52(13)$ & $1.0(1.2)$ \\
\hline Complex NP related & $645(27)$ & $11.3(2.1)$ & $839(42)$ & $12.7(2.6)$ \\
Complex NP unrelated & $647(26)$ & $10.6(2.2)$ & $832(36)$ & $12.3(2.4)$ \\
Difference & $-2(9)$ & $0.7(2.3)$ & $7(13)$ & $0.3(1.9)$
\end{tabular}

Note. Mean standard errors in brackets. Negative difference scores reflect phonological facilitation. $\mathrm{RT}=$ reaction times. NP $=$ noun phrase.

rates broken down by speed group, utterance format, and relatedness in the deadline condition. The results mirror those of the no-deadline condition: Despite the response deadline, there was still a significant difference between participants classified as fast and slow (main effect speed group: $\beta=0.043$, $S E=0.010, \quad t=4.2)$. Phonologically related distractors again decreased naming latencies compared to unrelated distractors (main effect relatedness: $\quad \beta=0.021, \quad S E=0.003, \quad t=7.3$ ). Utterance format and relatedness interacted $(\beta=0.024, S E=0.004, t=6.5)$, with a significant distractor effect in the bare noun condition $(\beta=0.045, S E=0.005, t=8.7)$, but not the complex NP condition (main effect relatedness: $\beta=0.002$, $S E=0.004, t=0.6$ ). Additionally, and somewhat counter-intuitively, participants were faster in naming complex NPs compared to bare nouns (main effect utterance format: $\beta=0.029, S E=0.010$, $t=3.0)$ None of the other effects were significant in the analysis of naming latencies $(t \mathrm{~s}<0.9)$.

Again, error rates were higher during the production of complex NPs (main effect utterance format: $\beta=0.696, S E=0.059, z=11.7, p<.001)$. Distractors phonologically related to the noun caused lower error rates than unrelated distractors (main effect relatedness: $\beta=0.149, S E=0.059, z$ $=2.5, p=.012)$. None of the other effects were significant in the analysis of error rates ( $p s>.175)$.

As for naming without a deadline, the distractor effect during bare noun naming was not correlated with the distractor effect during complex NP naming (slow speakers: $r=.08, p=.771$; fast speakers: $r=$ $.23, p=.399)$.

\section{Discussion}

Experiment 2 examined the effect of general naming speed on phonological advance planning. We contrasted fast and slow speakers in a speech production task both with and without a strict response deadline. We did not find effects of phonologically related distractors for complex NPs, and this held for fast and for slow speakers, and irrespective of the presence or absence of a response deadline. As in the study by Damian and Dumay (2007), a response deadline sped up naming latencies and effectively reduced inter- and intraindividual variability, as reflected in smaller standard errors. However, contrary to Damian and Dumay (2007), we found no phonological distractor effect for complex noun phrases both with and without a deadline. However, Damian and Dumay (2007) used a different complex NP format making a direct comparison with their results impossible. ${ }^{2}$

Our results do not replicate the difference in planning scopes between slow and fast speakers reported by Michel Lange and Laganaro (2014). However, when we look at the distractor effects per participant (see Figure 2 for an illustration of the individual effects), we can make the following three observations: (1) all but three participants showed a facilitation effect for the bare noun, both with and without a response deadline; (2) the distractor effects for the utterance-final element during 
complex NP production varied substantially,

Table 3

Mean naming latencies in milliseconds and error rates in percent of Experiment 2 for the deadline condition, broken down by speed group (fast vs. slow), utterance format (bare noun vs. complex noun phrase) and relatedness (phonologically related vs. unrelated to the target noun), aggregated by participants.

\begin{tabular}{lllll}
\hline & \multicolumn{3}{c}{ Fast speakers } & Slow speakers \\
\cline { 2 - 5 } & RT in ms & Errors in \% & RT in ms & Errors in \% \\
\hline Bare noun related & $508(9)$ & $3.5(0.7)$ & $566(9)$ & $3.6(1.6)$ \\
Bare noun unrelated & $558(7)$ & $5.7(1.1)$ & $611(6)$ & $5.2(1.6)$ \\
Difference & $-50(6)$ & $-2.3(1.0)$ & $-44(5)$ & $-1.6(0.7)$ \\
\hline $\begin{array}{l}\text { Complex NP related } \\
\text { Complex NP }\end{array}$ & $515(10)$ & $15.3(2.6)$ & $554(13)$ & $13.2(2.2)$ \\
unrelated & $513(10)$ & $17.2(3.0)$ & $551(15)$ & $14.6(2.5)$ \\
Difference & $2(5)$ & $-1.9(1.6)$ & $3(6)$ & $-1.4(2.4)$
\end{tabular}

Note. Mean standard errors in brackets. Negative difference scores reflect phonological facilitation. RT $=$ reaction times. NP $=$ noun phrase.

ranging from approximately $100 \mathrm{~ms}$ facilitation to $100 \mathrm{~ms}$ interference without a deadline, and from $40 \mathrm{~ms}$ facilitation to $60 \mathrm{~ms}$ interference with a deadline; and (3) the slope from the bare noun to the complex NP effects again, as in Experiment 1, was positive for all but five participants. We will return to these points in the General Discussion.

Additionally, one needs to bear in mind that in our case, the to-be-produced utterance was longer (i.e., four words) than in the study by Michel Lange and Laganaro (2014), but in both cases, the phonological activation of the final element was investigated. It is therefore possible that with our utterance format, we hit the final 'boundary' at which phonological advance planning is indeed possible, and differences between fast and slow speakers are only minuscule at this point of the utterance. We therefore investigated whether such differences may at least be partly visible across the naming latency distribution. To this end, we generated delta plots for both speed groups. In these plots, participants' naming latencies (aggregated by utterance format, phonological relatedness, and participant) are grouped in quintiles and plotted against the distractor effect (naming latencies of the related minus the unrelated condition) in each quintile on the $y$ axis. In other words, individual distractor effects can be examined in their magnitude and polarity in different time windows across the range of naming latencies. These plots allow - in an exploratory, post-hoc manner - to trace the time course of potential planning effects, and importantly, to compare them between speed groups and deadline conditions.

The left part of Figure 3 displays the distractor effect for the complex NP production without a response deadline. Fast speakers exhibit a smaller range of overall naming latencies $(\sim 500-850 \mathrm{~ms})$, whereas slow speakers' responses extend up to around $1100 \mathrm{~ms}$. When we look at the group-specific distractor effects as a function of quintile, another difference between the two groups emerges: Numerically, these effects are small for both fast and slow speakers, but the slow speakers display a larger variability with respect to magnitude and polarity of the distractor effect, whereas the fast speakers are grouped closer around 0ms across the naming latency distribution. This suggests that fast speakers 

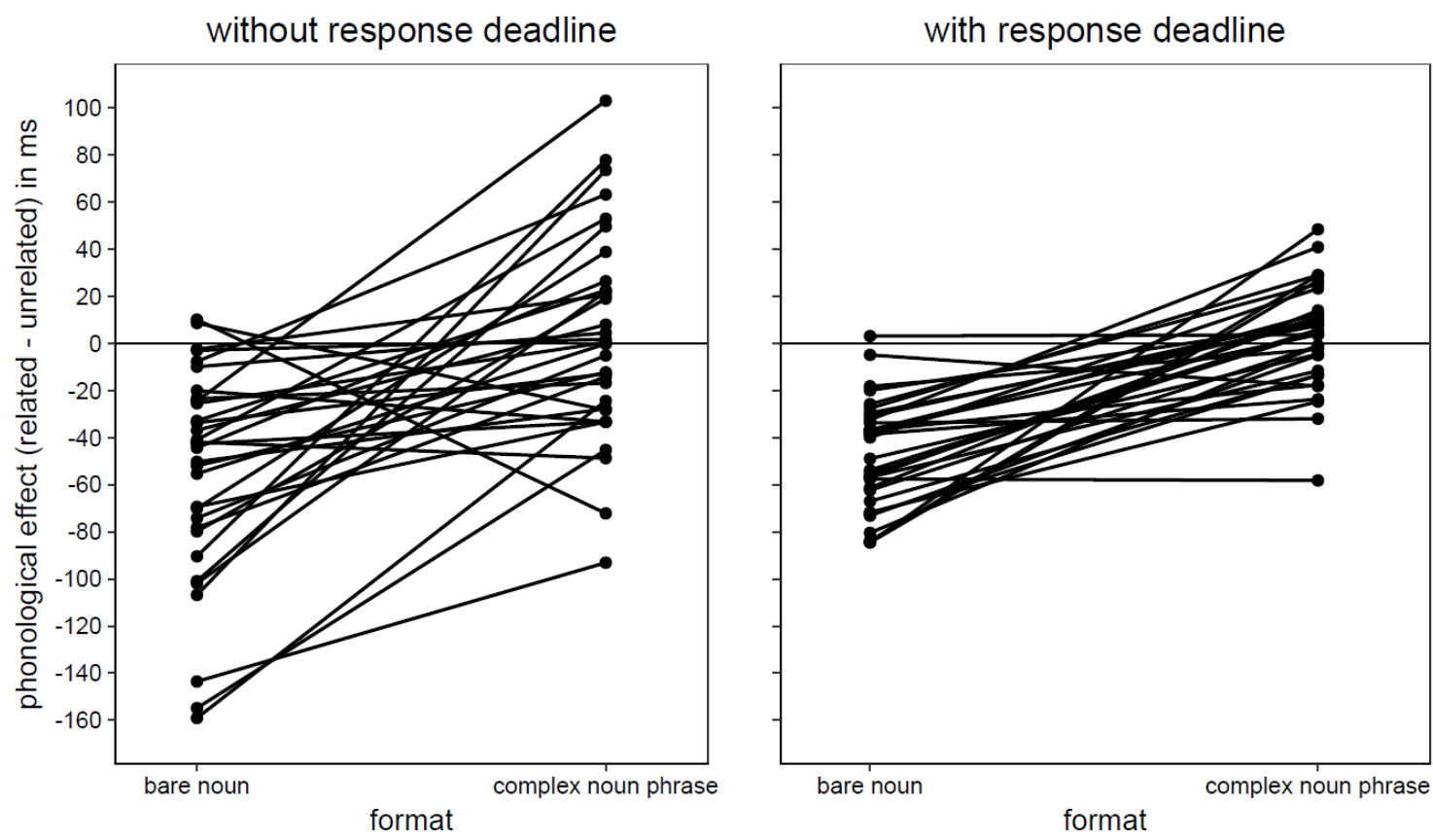

Figure 2. Individual distractor effects for the bare noun and the complex noun phrase conditions for Experiment 2, broken down for both deadline conditions. Negative values indicate facilitation from phonologically related distractors, and positive values indicate interference.

initiated their responses before having planned the entire utterance up to the phonological level. By contrast, slow speakers initially exhibit a descriptive interference effect from related distractors in the early time windows $(\sim 650-900 \mathrm{~ms})$, but this pattern reverses during the latest responses $(900-1100 \mathrm{~ms})$, resulting in an overall null effect obtained in the grand averages. In the presence of the response deadline (right part of Figure 3), the two groups converge. Overall, then, the delta plots provide tentative evidence for inter-individual differences of phonological advance planning in the production of multi-word utterances.

\section{General Discussion}

The current study investigated the potential influence of individual verbal working memory capacity (Experiment 1) and differences in general naming speed (Experiment 2) on phonological advance planning in complex NPs. In both experiments, we found faster naming latencies in the presence of target-related distractors when participants produced bare nouns (e.g., "aap" [monkey]), but no effect when participants produced complex NPs (e.g., "de kleine rode aap" [the small red monkey]). Also, there was no difference in distractor effects between WMC groups and between fast and slow participants, and this held irrespective of the presence or absence of a response deadline. When we follow the argumentation of earlier studies on phonological advance planning in complex utterances (e.g., Costa \& Caramazza, 2002; Damian \& Dumay, 2007; Michel Lange \& Laganaro, 2014), the conclusion to be drawn from the grand average null effects for complex utterances would have to be that speakers do not plan the utterance final word of complex utterances at the phonological level and that neither interindividual differences in working memory capacity nor general naming speed affect the phonological advance planning scope. However, when we look at the individual distractor effects (plotted in Figures 1 and 2), we do observe a striking consistency: during bare noun production, nearly all participants showed facilitation from related distractors, which is completely in line with the available studies in the literature. During complex NP production, however, we observed a vast interindividual variability of effects, ranging from facilitation effects over null effects to interference effects. Jescheniak et al. (2003) had proposed that the net effects observed for utterance final words result from a mixture of a facilitation component (due to facilitated phonological encoding of the target word) and an inhibitory component (due to a disturbance of the coding of the linear order of words in the utterance). From this perspective it would appear that in some of our speakers the 
facilitating component was dominant while in others

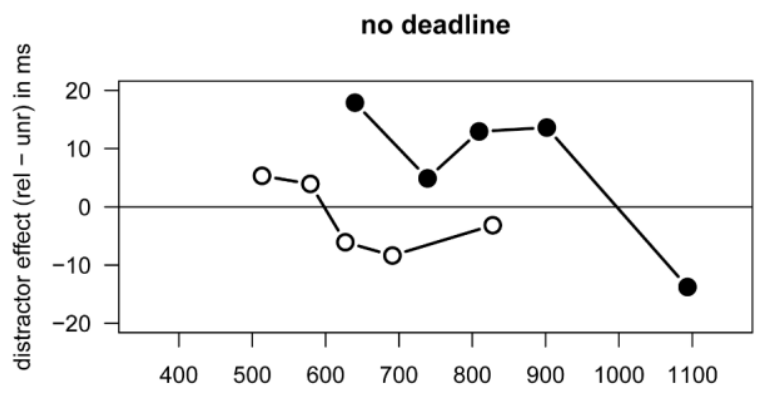

the inhibitory component was dominant and still in

deadline

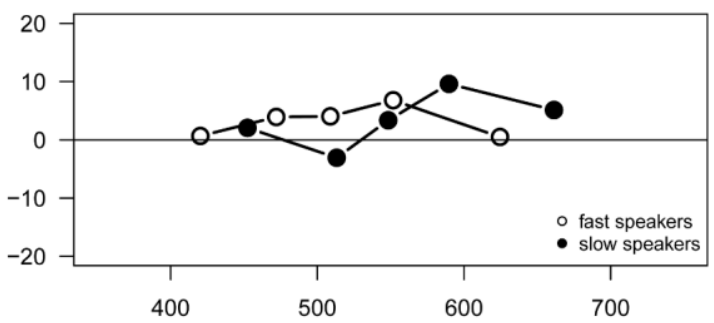

Figure 3. Delta plots for the distractor effect (phonologically related-unrelated, in ms) for the production of complex NPs, separated by deadline condition (naming without vs. with a response deadline) and individual naming speed (fast vs. slow). The $x$-axis plots the distribution of naming latencies (in ms) averaged across participants. The y-axis depicts the distractor effect. Negative values indicate facilitation and positive values interference from phonologically related distractors.

other speakers the two components cancelled each other out. The within-participant design of our study allowed us to examine whether the size of the facilitation effect in bare noun naming predicts the distractor effect in complex NP naming. When correlating the individual distractor effects (bare noun vs. complex NP) for the two experiments, however, we found no evidence that the two are associated. Therefore, the magnitude of the base priming effect as such does not explain the dominance of the facilitatory or inhibitory components contributing to the net-effect of related distractors However, what appears to be clear from the overall picture is that speakers do engage in phonological planning of the final element of complex noun phrases, albeit to different extents and potentially using different strategies. This also implies that only looking at the grand average results as an index of phonological advance planning (Tables 1 and 2) can be highly misleading.

A potential caveat of the current study is that utterance format was blocked throughout the experiments, such that participants always named the bare nouns first and the complex noun phrases in a subsequent block. As indicated in the introduction to Experiment 1, this was a conscious design decision with the goal of having a "phonological baseline effect" (bare noun naming) that is measured in the same way for all participants. Nevertheless, one could argue that part of the distractor effects or absence thereof - for the complex NP conditions might be contaminated by repetition effects. However, with the current design, potential effects of repetition are presumably negligible. Because we only had two distractor conditions (phonologically related vs. unrelated), participants had named the pictures only twice in the bare noun condition before turning to the complex NP condition (and six times for the deadline condition in Experiment 2). In a series of similar experiments which investigated phonological advance planning in subject-verbobject sentences, Klaus et al. (2017) had participants name the sentences eight times each before turning to the experimental blocks and still observed reliable phonological activation of the utterance-final element. This shows that the absence of a distractor effect during complex NP production in the present study cannot be attributed to repetition related processes.

The delta plots of Experiment 2 give us some additional - and admittedly exploratory - insight into phonological advance planning. They reveal a more variable advance planning scope for slow speakers along the naming latency distribution, whereas for fast speakers the scope of phonological advance planning does not depend on when they start speaking (see Figure 3). This does point towards some kind of involvement of naming speed in phonological advance planning in complex utterances. However, based on the current study, we cannot say whether this reflects specific planning strategies or is determined by individual cognitive factors. Imposing a response deadline did show that we can diminish these differences between fast and slow speakers, but this does not speak to the question why they exhibit differential planning patterns without time pressure.

In light of these findings, we would like to emphasise the need to look beyond grand averages of distractor effects when investigating phonological advance planning in complex utterances. Given the large variability between, but also within speakers, 


\section{DIFFERENCES IN PHONOLOGICAL ADVANCE PLANNING}

we propose to always include more detailed analyses which may provide a more fine-grained picture of inter- and intraindividual differences in the mechanisms underlying advance planning.

\section{Notes}

${ }^{1}$ Note that although such an extreme-group approach is not without criticism (Conway et al., 2005), we preferred it over an approach in which WMC is treated as a continuous variable for the present purposes. Given that such a direct investigation of the influence of WMC on phonological advance planning has not been done yet, we reasoned that looking at the extreme groups of the distribution as a first step might give a more direct indication of whether such a relationship exists at all, because differences should be most pronounced between the two groups. Only if such a dissociation emerges, a more detailed investigation including many more participants from the entire WMC distribution would be warranted to detect the specific relation between WMC and advance planning.

${ }^{2}$ The only study that used the same utterance format as the current study (i.e., determiner + size adjective + colour adjective + noun) was the one by Jescheniak et al. (2003), while all other studies used shorter formats. However, in the present experiments we used a fixed size-colour combination for a given picture across conditions (see Appendix A), while Jescheniak et al. systematically varied the visual attributes determining the target utterance within pictures across items . It might thus be possible that in our study, participants got accustomed to specific combinations of colour, size and picture, making the utterances more predictable and thus easier to produce. To rule out that this difference is responsible for the fact that Jescheniak et al did find distractor effects for utterance final elements while we did not, we conducted a control experiment with 32 additional participants, which was identical to Experiments 1 and 2 , but alternated the size and colour adjectives within pictures across conditions in the same way as in Jescheniak et al. Furthermore, we tested an additional SOA $(-100 \mathrm{~ms})$ because presenting the distractor at the same time as the target picture (SOA $0 \mathrm{~ms}$ ) might have been too late to actually unfold its effect sufficiently for the majority of the participants. However, we again only observed a significant interaction between distractor relatedness and utterance format $(\beta=0.033, S E=0.005, t=6.9)$, reflecting faster naming latencies in the presence of related distractors during bare noun production (related: 608ms, unrelated: 691ms; $\beta=0.063$, $S E=0.009, t=7.4)$, but not during complex NP production (related: $746 \mathrm{~ms}$, unrelated: $743 \mathrm{~ms}$; $\beta=0.002, S E=0.006, t=0.4)$.

\section{References}

Baayen, R. H., Davidson, D. J., \& Bates, D. M. (2008). Mixed-effects modeling with crossed random effects for subjects and items. Journal of Memory and Language, 59(4), 390-412. http://doi.org/10.1016/j.jml.2007.12.005

Baddeley, A. (2003). Working memory and language: an overview. Journal of Communication Disorders, 36(3), 189-208. Retrieved from http://www.ncbi.nlm.nih.gov/pubmed/1274266 7

Barr, D. J., Levy, R., Scheepers, C., \& Tily, H. J. (2013). Random effects structure for confirmatory hypothesis testing: Keep it maximal. Journal of Memory and Language, 68(3), 255-278. http://doi.org/10.1016/j.jml.2012.11.001

Bates, D., Mächler, M., Bolker, B., \& Walker, S. (2015). Fitting Linear Mixed-Effects Models Using lme4. Journal of Statistical Software, 67(1), $1-48$. http://doi.org/10.18637/jss.v067.i01

Conway, A. R. A., Kane, M. J., Bunting, M. F., Hambrick, D. Z., Wilhelm, O., \& Engle, R. W. (2005). Working memory span tasks: A methodological review and user's guide. Psychonomic Bulletin \& Review, 12(5), 769786. http://doi.org/10.3758/BF03196772

Costa, A., \& Caramazza, A. (2002). The Production of Noun Phrases in English and Spanish: Implications for the Scope of Phonological Encoding in Speech Production. Journal of Memory and Language, 46(1), 178-198. http://doi.org/10.1006/jmla.2001.2804

Damian, M. F., \& Dumay, N. (2007). Time pressure and phonological advance planning in spoken production. Journal of Memory and Language, $57(2)$,

195-209. http://doi.org/10.1016/j.jml.2006.11.001

Damian, M. F., \& Martin, R. (1999). Semantic and phonological codes interact in single word production. Journal of Experimental Psychology: Learning, Memory, and Cognition, 25, 1-18. 
Daneman, M., \& Merikle, P. M. (1996). Working memory and language comprehension: A metaanalysis. Psychonomic Bulletin \& Review, 3(4), 422-433. http://doi.org/10.3758/BF03214546

Hartsuiker, R. J., \& Barkhuysen, P. N. (2006). Language production and working memory: The case of subject-verb agreement. Language and Cognitive Processes, 21(1-3), 181-204. http://doi.org/10.1080/01690960400002117

Jaeger, T. F. (2008). Categorical data analysis: Away from ANOVAs (transformation or not) and towards logit mixed models. Journal of Memory and Language, 59(4), 434-446. http://doi.org/10.1016/j.jml.2007.11.007

Jescheniak, J. D., Schriefers, H., \& Hantsch, A. (2003). Utterance format affects phonological priming in the picture-word task: implications for models of phonological encoding in speech production. Journal of Experimental Psychology. Human Perception and Performance, 29(2), 441-54. http://doi.org/10.1037/0096-1523.29.2.441

Klaus, J., Mädebach, A., Oppermann, F., \& Jescheniak, J. D. (2017). Planning sentences while doing other things at the same time: effects of concurrent verbal and visuospatial working memory load. The Quarterly Journal of Experimental Psychology, 70(4), 811-831. http://doi.org/10.1080/17470218.2016.1167926

Klaus, J., \& Schriefers, H. (2016). Measuring verbal working memory capacity: A reading span task for laboratory and web-based use.

Meyer, A. S. (1996). Lexical Access in Phrase and Sentence Production: Results from PictureWord Interference Experiments. Journal of Memory and Language, 35(4), 477-496. http://doi.org/10.1006/jmla.1996.0026

Michel Lange, V., \& Laganaro, M. (2014). Intersubject variability modulates phonological advance planning in the production of adjectivenoun phrases. Frontiers in Psychology, 5, 43. http://doi.org/10.3389/fpsyg.2014.00043

Oppermann, F., Jescheniak, J. D., \& Schriefers, H. (2010). Phonological advance planning in sentence production. Journal of Memory and Language, 63(4), 526-540. http://doi.org/10.1016/j.jml.2010.07.004

Schnur, T. T. (2011). Phonological Planning during Sentence Production: Beyond the Verb. Frontiers in Psychology, 2, 319. http://doi.org/10.3389/fpsyg.2011.00319

Schnur, T. T., Costa, A., \& Caramazza, A. (2006). Planning at the Phonological Level during
Sentence Production. Journal of Psycholinguistic Research, 35(2), 189-213. http://doi.org/10.1007/s10936-005-9011-6

Schriefers, H. (1992). Lexical access in the production of noun phrases. Cognition, 45(1), 33-54. http://doi.org/10.1016/00100277(92)90022-A

Schriefers, H., de Ruiter, J. P., \& Steigerwald, M. (1999). Parallelism in the production of noun phrases: Experiments and reaction time models. Journal of Experimental Psychology-Learning Memory and Cognition, 25(3), 702-720. http://doi.org/10.1037/0278-7393.25.3.702

Schriefers, H., Meyer, A. S., \& Levelt, W. J. M. (1990). Exploring the time course of lexical access in language production: Picture-word interference studies. Journal of Memory and Language, 29(1), 86-102. http://doi.org/10.1016/0749-596X(90)90011-N

Schriefers, H., \& Teruel, E. (1999). Phonological Facilitation in the Production of Two-word Utterances. European Journal of Cognitive Psychology, 11(1), 17-50. http://doi.org/10.1080/713752301

Shao, Z., Roelofs, A., \& Meyer, A. S. (2012). Sources of individual differences in the speed of naming objects and actions: The contribution of executive control. The Quarterly Journal of Experimental Psychology, 65(10), 1927-1944. http://doi.org/10.1080/17470218.2012.670252

Smith, M., \& Wheeldon, L. (2004). Horizontal Information Flow in Spoken Sentence Production. Journal of Experimental Psychology: Learning, Memory, and Cognition, 30(3), 675-686. http://doi.org/10.1037/02787393.30.3.675

Wagner, V., Jescheniak, J. D., \& Schriefers, H. (2010). On the flexibility of grammatical advance planning during sentence production: Effects of cognitive load on multiple lexical access. Journal of Experimental Psychology: Learning, Memory, and Cognition, 36(2), 423440. http://doi.org/10.1037/a0018619

Wheeldon, L., Ohlson, N., Ashby, A., \& Gator, S. (2013). Lexical availability and grammatical encoding scope during spoken sentence production. The Quarterly Journal of Experimental Psychology, 66(8), 1653-1673. http://doi.org/10.1080/17470218.2012.754913

Zhao, L.-M., \& Yang, Y.-F. (2016). Lexical Planning in Sentence Production Is Highly Incremental: Evidence from ERPs. PLOS ONE, 
11(1), e0146359.

http://doi.org/10.1371/journal.pone.0146359 
Appendix A

Nouns, distractors, and size/colour adjectives used in Experiments 1 and 2.

\begin{tabular}{|c|c|c|c|c|}
\hline Target noun & $\begin{array}{l}\text { Related } \\
\text { distractor }\end{array}$ & $\begin{array}{l}\text { Unrelated } \\
\text { distractor }\end{array}$ & $\begin{array}{c}\text { Size } \\
\text { adjective }\end{array}$ & $\begin{array}{r}\text { Colour } \\
\text { adjective }\end{array}$ \\
\hline aap [monkey] & aad & miek & klein & rood \\
\hline auto [car] & ause & odig & klein & groen \\
\hline berg [mountain] & belk & pijt & klein & groen \\
\hline bloem [flower] & bloer & eest & klein & rood \\
\hline eekhoorn [squirrel] & eemkirt & spiemer & groot & blauw \\
\hline eend [duck] & eest & bloer & klein & rood \\
\hline emmer [bucket] & ellig & ause & klein & rood \\
\hline fles [bottle] & flem & holk & klein & rood \\
\hline handschoen [glove] & hampruig & vlimsel & klein & rood \\
\hline heks [witch] & helg & junt & klein & rood \\
\hline hond $[\mathrm{dog}]$ & holk & kig & groot & blauw \\
\hline jurk [dress] & junt & rilm & klein & groen \\
\hline kaas [cheese] & kaam & stoen & groot & bruin \\
\hline kikker [frog] & kifsel & tremsel & groot & blauw \\
\hline kip [chicken] & kig & flem & groot & blauw \\
\hline koffer [suitcase] & kosje & spijmul & groot & bruin \\
\hline mier [ant] & miek & staag & groot & bruin \\
\hline munt [coin] & murg & wops & klein & rood \\
\hline ober [waiter] & odig & schinsler & klein & groen \\
\hline pijl [arrow] & pijt & helg & & bruin \\
\hline ridder [knight] & rissel & wolper & klein & groen \\
\hline riem [belt] & rief & aad & klein & groen \\
\hline rits [zipper] & rilm & $\operatorname{tad}$ & groot & bruin \\
\hline rolstoel [wheelchair] & ronser & kifsel & groot & blauw \\
\hline schildpad [turtle] & schinsler & ronser & groot & bruin \\
\hline spiegel [mirror] & spiemer & vober & groot & bruin \\
\hline spijker [nail] & spijmul & kosje & groot & bruin \\
\hline start [tail] & staag & rief & groot & blauw \\
\hline stoel [chair] & stoen & kaam & groot & blauw \\
\hline tas [bag] & $\operatorname{tad}$ & wons & klein & rood \\
\hline trechter [funnel] & tremsel & hampruig & klein & groen \\
\hline vlinder [butterfly] & vlimsel & ellig & klein & groen \\
\hline vogel [bird] & vober & eemkirt & klein & groen \\
\hline wolk [cloud] & wons & murg & groot & bruin \\
\hline worst [sausage] & wops & belk & groot & blauw \\
\hline wortel [carrot] & wolper & rissel & groot & blauw \\
\hline
\end{tabular}

Note. Translations of the target nouns are given in brackets. Groot $=$ big; klein $=$ small; blauw $=$ blue; groen $=$ green; rood $=$ red; bruin $=$ brown . 